\title{
Observational Study of Pulmonary Embolism Patients in Shahid Gangalal National Heart Centre
}

\author{
Rauniyar $\mathrm{B}^{*}$, Adhikari $\mathrm{CM}^{*}$, Rajbhandari $\mathrm{R}^{*}$,Limbu $\mathrm{YR}^{*}$,Malla $\mathrm{R}^{*}$, Sharma $\mathrm{R}^{*}$, Rajbhanda- \\ ri $\mathrm{S}^{*}$,Sharma $\mathrm{D}^{*}$,Maskey $\mathrm{A}^{*}$, Singh S.K ${ }^{*}$,Prajapati $\mathrm{D}^{*}$,Joshi $\mathrm{D}^{*}, \mathrm{~K}^{*}$.C MB* \\ *Department of Cardiology, Shahid Gangalal National Heart Centre, Bansbari, \\ Kathmandu
}

\section{ABSTRACT}

Pulmonary thromboembolism (PE) is a relatively common cardiovascular emergency, with a significant mortality and morbidity often precipitated by deep venous thrombosis (DVT).It is considered as the third most common cardiovascular disorder after Acute Coronary Syndromes (ACS) and Stroke. This study aims to find out the clinical characteristic of Nepali PE patients. We hope that it can be helpful in the early recognition and management of PE patient. Eleven consecutive patients PE who were diagnosed based on CT report were studied. Predisposing factors were same as the other international study. Among eleven patients three were diagnosed as high risk pulmonary embolism, others were intermediate risk. Shortness of breath, chest discomforts are the commonest symptom. Echo revealed Dilated RA and RV were present in all patients. All the patients were treated with Heparin/Low molecular Heparin. Among the three high risk PE patients only one patient was treated with Thrombolytic therapy because of the missed diagnosis. The diagnosis is the most difficult and tricky part in the management of $\mathrm{PE}$ as treatment is the easiest part. Early suspicion is the most important part in the management of PE as symptom and sign are non-specific.

Correspondence:

Chandra Mani Adhikari

Shahid Gangalal National Heart Centre, Bansbari,Kathmandu,Nepal

Tel: 00977-1-4371322, 00977-1-4371374.

Fax: 00977-1-4371123

Email:topjhap@hotmail.com 


\section{INTRODUCTION}

Pulmonary thromboembolism (PE) is a relatively common cardiovascular emergency ${ }^{1}$, with a significant mortality and morbidity often precipitated by deep venous thrombosis (DVT).It is considered as the third most common cardiovascular disorder after Acute Coronary Syndromes (ACS) and Stroke, with 300000 hospitalizations and 50000 deaths annually in US and as many as 200000 deaths annually in the European Union. 2,3,4 The true incidence of PE is unknown. Because its many non-specific clinical features it is one of the most difficult diagnostic challenges in all of medicine. ${ }^{5,6}$ It has been reported, that only one third of patients dying with PE have a correct ante mortem diagnosis. ${ }^{5}$ The diagnosis was never even made in most of these patients ante mortem. Although some patients die despite a correct and early diagnosis, Mortality is fourfold to six fold greater in those patients in whom the diagnosis was missed. Thus, there is continued interest in the accuracy of the clinical ante mortem diagnosis of PE. ${ }^{7}$ Though small number of patient population we are trying to find out the clinical characteristic of Nepali PE patients. We hope that it can be helpful in the recognition and management of $\mathrm{PE}$ patient.

\section{METHODS}

Eleven consecutive patients who were treated in our cardiac center for PE were studied. The diagnosis was confirmed by CT. All the patient data in this study were obtained from hospital registry.

\section{RESULTS}

Clinical characteristics of the patients included in this study are shown in the table. The age range was between 31- 68years. There were 8 male and 3 female patients. Among eleven patients three were diagnosed as high risk pulmonary embolism, eight were intermediate risk.

$\begin{array}{ll}\text { Characteristics } & \mathbf{n = 1 1} \\ \text { Age } & 31-68 \text { years } \\ \text { Male/Female } & 8 / 3 \\ \text { High risk PE } & 3 \\ \text { Intermediate risk PE } & 8\end{array}$

About the predisposing factors two have facture bone; cancer in one, recent surgery in one, remaining three patient don't have any predisposing factor.

$\begin{array}{ll}\text { Predisposing factor } & \\ \text { Cancer } & 1 \\ \text { Recent Surgery } & 1 \\ \text { Bone fracture } & 2\end{array}$

\section{CLINICAL PRESENTATION}

Nine of these patients presented to the ER. Nine cases were referred cases from other hospital with the diagnosis of ACS, only one patient was diagnosed as pulmonary embolism by the referring hospital. One patient presented to our ER and suspected to have PE on ECHO finding. Shortness of breath was present in all of them, chest discomfort in seven patients and syncope in three patients. Three patient presented in Cardiogenic shock. Sinus tachycardia was present in five patients.SPO2 was less than $90 \%$ in six patients. Troponin I was positive in just a single patient.

\section{ECG}

ECG finding of the patient are as follows:

\begin{tabular}{lc} 
ECG Finding & No of patients \\
\hline T waves negative in V1-V4 & 8 \\
S1 Q3 T3 pattern & 2 \\
Sinus tachycardia & 5 \\
Complete/incomplete RBBB & 2 \\
Normal & 3
\end{tabular}

\section{Chest X-ray}

Hampton humps in one patient all others have unremarkable finding in chest $\mathrm{X}$-ray.

\section{Echocardiography}

Dilated RA and RV were present in all patients.

\section{Treatment}

All the patients were treated with Heparin/Low molecular Heparin.

Among the three patients who were diagnosed as high risk pulmonary embolism and was supposed to be treated with Thrombolytic therapy only one patient was treated with it. Two high risk pulmonary embolism patients did not receive such therapy, as we could not diagnose them when they presented to us. They were treated in line of ACS initially. 


\section{Prognosis}

All the patients were discharged on Warfarin after few days of hospital stay.

\section{DISCUSSION}

This study is the first observational study on PE patients. As in the developed countries diagnosis of $P E$ is usually delayed and missed. To make a confirmatory diagnosis of $\mathrm{PE}, \mathrm{CT}$ is needed which is not only expensive but also not easily available. As the symptoms of PE are non-specific and confirmatory test not easily available unless the clinical feature are clearly in favor of PE then only patient are sent for the confirmatory test. This may be the reason behind delayed diagnosis. Most of the patients in our study were referred cases with the diagnosis of ACS and later diagnosed as PE; this suggests that earlier suspicion and diagnosis of the disease would largely help in the early management of the disease.

In our population the predisposing factors are same like those in other international studies. Sudden onset of shortness of breath was the most common symptom in our patient like in other international studies ${ }^{8}$. Chest discomfort along with shortness of breath was the common presenting symptoms in our patient. Though these symptoms are not specific but we consider that these symptoms should make us suspicious of $P E$ unless explained otherwise. When we consider the usefulness of ECG in the diagnosis of $P E$, ECG findings in these patients are usually non-specific and many times they are suggestive of ACS which may be the reason why most of the patients were referred with the diagnosis of ACS. Though simple investigation such as chest radiography and ECG are often inadequate in the diagnosis but they are really useful in the evaluation of PE. ${ }^{9,10} \mathrm{~S} 1 \mathrm{Q} 3 \mathrm{~T} 3$ pattern is considered as the most specific ECG changes of PE but as in other studies they are not sensitive. Negative $T$ waves in Leads V1-V4 a sign of RV strain is com- mon in our study population, though this pattern can be a normal variant and an ECG changes of ACS. But it can help us to make a suspicion when the clinical findings are suggestive of PE.

It is considered that bedside transthoracic echocardiography can provide some information to substantiate the diagnosis, ${ }^{11}$ When ECHO finding of dilated RA and RV are considered along with predisposing factors, symptom and ECG findings, we can easily come close to the diagnosis of PE, as we have done it many times in our study. Among these eleven patients one of the patients was confirmed as PE, only on clinical background with the help of ECG and ECHO and managed with Thrombolytic agent without the report of CT. This is the first time we have successfully thrombolysed a PE patient without a confirmatory CT report in our center. This practice was done on the base of the "ESC Guidelines on the diagnosis and management of acute pulmonary embolism".

The diagnosis is the most difficult and tricky part in the management of PE as treatment is the easiest part. Thrombolytic therapy is the first-line treatment in patients with high-risk PE presenting with cardiogenic shock and/or persistent arterial hypotension ${ }^{8}$. All the others patient can easily be managed with Heparin followed by warfarin with very good prognosis.

\section{CONCLUSION}

PE is a common cardiovascular emergency. Diagnosis of PE is tricky because of non-specific sign and symptom. Shortness of breath and chest discomfort is the common symptoms. Though ECG and chest $\mathrm{x}$-ray has minimal role in the diagnosis but they can help us to make us suspicious about the diagnosis. ECHO can provide important information in the diagnosis of PE. Suspicion is the most important part to come to the diagnosis of PE. 


\section{REFERENCES}

1. Roy PM, Meyer G, Vielle B, Le Gall C, Verschuren F, Carpentier F et al. Appropriateness of diagnostic management and outcomes of suspected pulmonary embolism. Ann Intern Med 2006;144:157-164.

2. Bell WR, Simon TL. Current status of pulmonary thrombo-embolic disease: pathophysiology, diagnosis, prevention and treatment. Am Heart J 1982; 103:239 -262.

3. Lilienfeld DE, Chan E, Ehland J, et al. Mortality from pulmonary embolism in the United States: 1962 to 1984. Chest 1990; 98:10671072.

4. Stavros $\mathrm{V}$ Konstantinides.Acute pulmonary embolism revisited: 2008 84: 651-658 Postgrad Med J).

5. Goldhaber SZ, Hennekens CH, Evans DA, et al. Factors associated with correct antemortem diagnosis of major pulmonary embolism. Am J Med 1982; 73:822- 826

6. Anderson FAJ, Goldberg RJ, Hosmer DW, et al. A popula-tion-based perspective of the hospital incidence and case-fatality rates of deep vein thrombosis and pulmonary embolism:the Worcester DVT Study. Arch Intern Med 1991;151:933-938.

7. Lilibeth A. Pineda, Vasanthakumar S. Hathwar and Brydon J. B. Grant Clinical Suspicion of Fatal Pulmonary Chest:2001;120;791-795.

8. ESC Guidelines on the diagnosis and management of Acute pulmonary embolism. European heart journal(2008)29,2276-2315.

9. Hyers TN, Hull RD, Weg JG. Antithrombotic therapy for venous thromboembolic disease. Chest 1995; 108:335S.

10. Janata-Schwatczek K, Weiss K, Riezinger I, Bankier A, Domanovits H, Seidler D. Pulmonary embolism II. Diagno-sis and treatment. Semin Thromb Hemostas 1996;22:33-52.

11. Come PC. Echocardiographic evaluation of pulmonary embolism and its response to therapeutic interventions. Chest 1992;101(suppl):151-62. 